\title{
Lively Social Space, Well-Being Activity, and Urban Design: Findings from a low-cost community-led public space intervention
}

\author{
Jamie Anderson ${ }^{1}$, Kai Ruggeri ${ }^{1}$, \\ Koen Steemers ${ }^{1}$, and Felicia Huppert ${ }^{1}$
}

\begin{abstract}
Empirical urban design research emphasizes the support in vitality of public space use. We examine the extent to which a public space intervention promoted liveliness and three key behaviors that enhance well-being ("connect," "be active," and "take notice"). The exploratory study combined directly observed behaviors with self-reported, before and after community-led physical improvements to a public space in central Manchester (the United Kingdom). Observation data ( $\mathrm{n}=$ 22,956 ) and surveys (subsample $=212$ ) were collected over two 3-week periods. The intervention brought significant and substantial increases in liveliness of the space and well-being activities. None of these activities showed increases in a control space during the same periods. The findings demonstrate the feasibility of the research methods, and the impact of improved quality of outdoor neighborhood space on liveliness and well-being activities. The local community also played a key role in conceiving of and delivering an effective and affordable intervention. The findings have implications for researchers, policy makers, and communities alike.
\end{abstract}

Keywords: liveliness, well-being, neighborhood, population intervention, urban design

\footnotetext{
${ }^{1}$ University of Cambridge, Cambridge, UK Corresponding Author: Jamie Anderson, Department of Architecture, University of Cambridge, 1-5 Scroope Terrace, Cambridge, CB2 1PX.

Email: anderson.jamie@gmail.com
} 


\section{Introduction}

Urban design literature provides evidence of the importance of attractive public space in supporting a sense of safety, pleasurable experiences (Childs, 2004; Cooper Marcus \& Francis, 1998), public life (Gehl, 2004), or what Whyte referred to as "city moments" where strangers enjoy a shared experience (Whyte, 1980). Mehta $(2007,2013)$ contends that the support of high-quality urban spaces may extend to the promotion of social and psychological health in modern communities. As noted by previous scholars (e.g., Jacobs, 1961; Lynch, 1984; Oldenburg, 1981; Tibbalds, 1992), good urban spaces have the ability to support and promote all public and communal life, which is an essential counterpart to our private home and work spaces (Mehta, 2007).

Further reasoning that high-quality urban design may represent effective support for well-being is provided by recent physical activity research. For example, physical activity interventions targeted at individuals, such as physical education, can be effective in promoting activity in some circumstances. However, their use has not been associated with a discernible increase in population activity levels (Dombrowski, Knittle, Avenell, Araújo-Soares, \& Sniehotta, 2014; Marteau, Ogilvie, Roland, Suhrcke, \& Kelly, 2011). Population-level urban design intervention, such as the provision of salient outdoor neighborhood opportunities and public space, may offer constructive insight. In particular, the socio-ecological (Bronfenbrenner \& Morris, 2007) and reflective-impulsive behavioral (Strack \& Deutsch, 2004) models suggest that targeting interventions at the social and physical environment may be more productive than at the individual level.

The establishment of clear links between the design of urban public space and the promotion of well-being activities would be meaningful. A recent comparison of mental health among European countries found that $19 \%$ of the U.K. population, the vast majority of whom live in urban areas (Office for National Statistics [ONS], 2011; United Nations [UN], 2016), has mental health positive enough to be described as flourishing. However, this does not compare favorably with the highest recorded prevalence of $42 \%$ among the Danish population 
(Huppert \& So, 2013), most of whom also live in urban areas (UN, 2016). Despite there being theoretical reasons linking urban design and the prevalence of well-being, in particular via the help or hindrance of communal activities, to date, most empirical public space research focuses on street liveliness or the pursuit of essential and optional activities outdoors, duration of stay (Gehl, 2004), and number of people engaged in social activity (Gehl, 2004; Mehta, 2007). The targeting of these activities and increasing liveliness in most public spaces is a crucial design goal in itself. However, the understanding of potential links between public space design and well-being represents a substantial gap in knowledge (Anderson, Ruggeri, Steemers, \& Huppert, in press; Ballas, 2013; Burton, 2015).

Our study contributes to existing research on liveliness and represents a step toward the linking of public space design and key wellbeing proxy measures. To understand how to improve well-being, a behavioral approach involving the Five-Ways to Well-being (Aked, Thompson, Marks, \& Cordon, 2008) represents the key starting point of this study.

\section{The Five-Ways}

The Five-Ways embody several of the most important actions we can take to improve our well-being and were derived from the sizable body of evidence gathered in the Foresight Project on Mental Capital and Well-being, published by the U.K. Government's Office for Science (GOS) in 2008. The Five-Ways approach was authored by the New Economics Foundation (nef) as accessible evidence-based actions for individuals to improve their personal well-being, or behaviors that organizations, communities, practitioners, and policy makers may encourage and support (Aked \& Thompson, 2011).

The Five-Ways approach has been taken up internationally as "downstream" (i.e., individual level) and/or "upstream" (i.e., environment level) interventions. These countries include Australia, Croatia, Holland, Iceland, Ireland, Japan, New Zealand, Norway, the United Kingdom, and the United States, and has been translated into a further six languages including Nepali, Chinese, Farsi, Somali, Amharic, 
and Arabic (nef, 2016). Examples of Five-Ways intervention at the individual level include mental health campaigns and personal development in schools. Environment-level instances include ensuring the strategic importance of the Five-Ways within public health strategies, programming of adult education, and streetscape assessment (Aked \& Thompson, 2011; nef, 2016). The Five-Ways are a priority topic in the United Kingdom, forming a critical emphasis of ongoing What Works Well-Being (2016) research. In the United States, New York State Department of Health has adopted the Five-Ways for their Prevention Agenda (2013-2018), the State's Community Health Improvement Plan for local action to improve the health of New Yorkers (nef, 2016).

Because the focus of this study is observed activity and public space design, we focused on the first three of the Five-Ways as they are particularly relevant, namely, Connect, Be Active, and Take Notice. The remaining two, Keep Learning and Give, are less easy to observe and measure in the context of people using outdoor space. Accordingly, we examined these three behavior types (the Three-Ways):

1. Connecting with other people, whether familiar or strangers (e.g., talking and listening);

2. Engaging in physical activity (e.g., competitive or casual ball games) and;

3. Taking notice or being aware of one's external environment (e.g., watching wildlife).

The above examples conceal the richness of the categories, and a more detailed description of these activities is provided by Aked et al. (2008). To capture the Three-Ways in public space, we adapted simplified measures, as limited by our principal method: behavior observation.

It is established that quantity and quality of individual social connections are critically correlated with subjective well-being (SWB; Dolan, Peasgood, \& White, 2008; GOS, 2008; Helliwell \& Putnam, 2005) and physical health (Krogh, Nordentoft, Sterne, \& Lawlor, 2011). Social relations include interaction with people close to us, such as friends and family, sometimes referred to as "thick ties," and more fleeting 
interaction with strangers and acquaintances, or "thin ties" (GOS, 2008; Halpern, 2010; Helliwell \& Putnam, 2005;). Being physically active is associated with higher SWB, a reduction in symptoms of mental illness, and the prevention of a range of chronic physical diseases (Krogh et al., 2011; Lee et al., 2012; Sofi et al., 2010).

Taking Notice (or mindfulness) has been strongly linked to higher SWB and fewer negative symptoms such as anxiety or depression (Chambers, Gullone, \& Allen, 2009; Hofmann, Sawyer, Witt, \& Oh, 2010; Tang, Yang, Leve, \& Harold, 2012). Mindfulness is the intentional, accepting, and non-judgmental focus of one's attention on the emotions, thoughts, and sensations occurring in the present moment (Paulson, Davidson, Jha, \& Kabat-Zinn, 2013). It involves Taking Notice of one's internal and external environments, and it is recommended that practice be taken up via formal training such as Mindfulness-Based Stress Reduction (MBSR). Within our study, we concentrate on individuals' external awareness (Taking Notice) of their external physical and social environments, as it was not possible to detect users' awareness of internal environments.

In short, there is no lack of evidence showing why Connecting, Being Active, and Taking Notice are important for high SWB. These activities are used as proxy measures within our study, as they are considered strong intermediary indicators of well-being (Aked et al., 2008; GOS, 2008). Keep Active is also a strong proxy for physical health (Centre for Disease Control and Prevention [CDC], 2015; Lee et al., 2012).

\section{Urban Design and the Three-Ways}

The urban design literature is strongest with regard to the dimension $\mathrm{Be}$ Active. Cross-sectional evidence suggests several urban design features are associated with overall physical activity, walking, and cycling (Bauman \& Bull, 2007; Kaczynski \& Henderson, 2007; Wendel-Vos, Droomers, Kremers, Brug, \& Van Lenthe, 2007). However, these studies may be susceptible to bias such as selective migration of residents (McCormack \& Shiell, 2011). There is also a lack of robust causational evidence gleaned from quasi-experiments and longitudinal studies, 
particularly in relation to large-scale environmental change (Mayne, Auchincloss, \& Michael, 2015).

Drawing on an adapted version of a Cochrane Risk of Bias Assessment Tool: for Non-Randomized Studies of Interventions (ACROBAT-NRSI), built environment natural experiment evaluations undertaken to date were found to be either critically or seriously biased (Benton, Anderson, Hunter, \& French, in press) and most research was carried out in the United States or Australia. Two further recent reviews also concluded that there is shortage of strong research, with the strongest insights provided for improved active transport infrastructure (Hunter et al., 2015; Mayne et al., 2015), or the combination of physical and psycho-social interventions (Hunter et al., 2015). Interventions involving improved quality of public space targeted at increased physical activity uncovered mixed findings. The positive results of significance to our study involved (a) the establishment of a new park in Australia (Veitch, Ball, Crawford, Abbott, \& Salmon, 2012), (b) the greening of vacant urban land in the United States (Branas et al., 2011), and (c) the introduction of three pocket parks, also from vacant and undesirable urban parcels in the United States (Cohen et al., 2014).

Evidence with regard to Connect activities and public spaces is not as encouraging and remains even more inconclusive. It is likely that some of the above successful interventions may have positively affected social activities, as well as physical activity. However, in most cases, these specific behaviors are not recorded and the same is true of most urban design studies (Cattell, Dines, Gesler, \& Curtis, 2008; Holland, Clark, Katz, \& Peace, 2007; Mehta, 2007). Where examples exist, most represent cross-sectional comparison of plazas, with some studies that focus on residential streets and spaces. In particular, these studies highlight the importance of walkable (i.e., pleasant and easy to use) streets in supporting social capital, which includes positive interactions with neighbors (Wood et al., 2008) and sense of community. Both these outcomes are likely to arise, in part, because of sustained local Connect activities (Lund, 2002).

The literature emphasizes superficial contacts between neighborhood members and perceived cohesion within the 
neighborhood, both of which have been found to be influenced by the availability of green and public space (De Vries, 2010; Maas, Dillen, Verheij, \& Groenewegen, 2009). Partial confirmation is provided by randomized studies, revealing that residents living next to green spaces reported more social activities and had more visitors, knew more of their neighbors, and had a stronger sense of belonging (Coley, Sullivan, \& Kuo, 1997; Kuo \& Sullivan, 1998). Although the number of studies is limited, validity of measures remains unclear, and causal mechanisms remain to be tested, it is proposed that high-quality outdoor neighborhood space provision encourages and attracts social interaction. This support may, with time, nurture strong ties, whereby social cohesion acts as a mediator (Blokland-Plotters, 2006; Cattell et al., 2008).

With regard to Take Notice, the authors are unaware of confirmation that public space users regularly anchor themselves in the present, drawing on visual, symbolic, audible, olfactory, or tactile information. Qualitative research suggests that users may be at least temporarily aware of their social environment. For example, Whyte's study of the Social Life of Small Urban Spaces showed that users appeared to take pleasure in the act of "people watching" and enjoyed listening to water features such as plaza fountains (Whyte, 1980). However, this remains an under-researched area, and in particular, it is unclear whether high-quality public space that affords pleasant sensory information may facilitate sustained Take Notice activities.

In sum, encouraging Be Active and, more tentatively, Connect and Take Notice built environment research highlights public space design as a worthwhile subject for further cross-sectional and natural experiment scrutiny.

\section{Key Challenges Addressed and Research Questions}

The absence of adequate urban design interventions studies is, in large part, due to the difficulty of using this research design in real world settings, which raises several scientific and evaluative challenges. For example, problems include matching research timetables with the regeneration or public space investment timelines, recruiting with 
speed to conduct a baseline assessment ahead of the intervention, and measuring confounding variables and levels of exposure (Hunter et al., 2015). Furthermore, the feasibility of a recent U.K. natural experiment pilot study was hampered by low response rates and highly inconsistent support from gatekeepers such as developers, estate agents, and housing associations (Ogilvie et al., in press).

Evaluations of public space interventions are complex, comprised of multiple interacting factors at the individual, community, and population levels. They need to be scientifically robust yet flexible enough to cope with unpredictable implementation, a changing environment and low participation rates-which cannot be controlled by the researcher (Hunter et al., 2015). This type of research incurs considerable costs and may require the use of unobtrusive measures. As a guide to achieving rigor and in light of practical constraints, the U.K. Medical Research Council (MRC) set out that, at a minimum, natural experiments should include the specification of a-priori hypotheses, clear definitions of target populations, explicit sampling criteria, and the use of valid and reliable outcome measures (Craig et al., 2012). More recently, Hunter et al. (2015) set out further recommendations such as the need for longer-term follow-up post intervention, adequate control groups, sufficiently powered studies, and consideration of the social environment.

Our exploratory study built on existing literature via a natural experiment that is informed by the above recommendations, representing a move toward improved research standards. We outline four key domains of further exploration within our discussion.

Our study design sought to test the following five hypotheses, expressed here as questions:

1. Do low-cost interventions to an outdoor public space increase the number of general users?

2. Do the interventions encourage users to stay longer?

3. Are the number of Three-Ways activities boosted among users?

4. Our research also paid attention to the individual small-scale public space features improved, such as the provision of public art, seating and WiFi, whether surfaces are green or hard- 
landscaped, and type of vegetation. Our study therefore also asked the following question:

5. Which features of outdoor space improvement are most strongly associated with the Three-Ways among users?

To address these questions, an approach was taken whereby behavior was observed directly as residents went about their local dayto-day outdoor activities. The behaviors were mapped so that results can be more clearly related to specific small-scale design characteristics, such as public art and street furniture. We also spoke to a subset of these users about their perceptions of the space. These insights can be tested further by researchers and practitioners in the future.

\section{Method}

The study used a mixed-methods approach comprising survey measures and detailed observations, in a natural experiment comparing matched treatment and control spaces. The experiment was comprised of a repeated cross-sectional rather than longitudinal study, as the exact same public space users were not tracked before and after the intervention.

The study took place in the city center of Manchester (the United Kingdom), in the Northern Quarter (NQ) of the city (see online supplemental appendix). Data from Public Health England (PHE), and the ONS National Well-being Measurement, show lower levels of wellbeing in Manchester compared with the United Kingdom and the NorthWest as a whole (ONS, 2013; PHE, 2013b). In addition, Public Health England's 2013 profile shows Manchester's physical health to be generally worse than the English average (PHE, 2013a).

The NQ is an area of Manchester city center, situated to the north of Piccadilly Gardens and to the south of Ancoats and centered around Oldham Street (Manchester City Council [MCC], 2003; see online supplementary materials). This neighborhood is one of the most vibrant and historically rich areas of Manchester. Today, the NQ is known for independent stores, creative industries, entertainment venues, cafes, and bars (Kellie, 2010). Until the 1990s and with the exception of a small amount of social housing, the NQ was not designated as a residential 
area. In recent years, however, the NQ has become a popular place to live, showing a substantial increase in residential community.

\section{Site Selection}

The treatment space was selected using a purposive sampling approach. This process was based on the need for intervention research and determining probable causes. Given the economic downturn at the time the study was undertaken, finding a public space improvement project that matched the funding timescales was difficult, despite advertising nationally via relevant institutional websites, forums, and industrial networks. From a list of three possible alternatives across the United Kingdom, the treatment space was chosen because,

1. The local community had expressed interest in imminent improvement of the space;

2. The site was owned outright by the Local Authority (MCC);

3. MCC planners had approved of intervention work in the space in principle; and

4. The projected project deadlines fell within the available data collection time window.

A rigorous approach was taken to the selection of the control space to ensure that it was well matched with the treatment space, in urban design terms. Both spaces are north-facing and overshadowed by buildings that replaced Victorian warehouses in the 1960s (Figures 1 and 2). The control space is very similar as it was created using the same materials, is situated along a road with comparable levels of vehicle movement (Department for Transport [DfT], 2013) and footfall, and provides little or no positive ground floor interface with the space. Further details of matching between the two spaces are provided in online supplemental appendix.

\section{Community Workshop and Implementation}

Ahead of the primary data collection, a workshop was held in June 2011, at a local venue and was facilitated by experienced urban design practitioners. The participants $(n=19)$ were provided with information describing the nature and purpose of the project and recruited via local 
community forums, and an MCC local resident database. The aim of the workshop was to provide an evaluation of existing local outdoor resources (e.g., parks and squares) and to enable participants to generate possible solutions to improve any shortcomings that might influence users and well-being behaviors. Further details of the workshop and collaboration can be found elsewhere (Anderson \& Baldwin, in press).

Several interventions suggested in the workshop were chosen for implementation on the basis of urgency, affordability, and likelihood of approval from the Local Authority. The community implemented the improvements, undertaking imaginative fundraising and, at times, hard physical labor. Interventions were completed before post-intervention data collection in July 2012.

The improvements, which cost approximately $\$ 20,000$ in total, included the introduction of an outdoor exhibition space comprising two types of ecologically based public art. The first involved the creation of a "Bug Hotel-Strip," which was named "Louse Vegas." The main purpose of the hotel-strip was to accommodate endangered native invertebrates (e.g., beetles and bees). Signs depicting hotel names and invertebrate information were also constructed and hung on or adjacent to the hotels. The second strand involved commissioning a local artist to create an environmentally oriented mural, which depicted a cartoon woodland setting (example images are provided in the online supplemental appendix). Additional work included the installation of a free high-speed WiFi service, shade-tolerant planting, an inner-city lawn, vegetation management, recycled seating, painting, and general cleaning.

Care was taken to avoid media coverage of the project, both at its inception and during the period of observations, so that expectations would not contaminate the results.

\section{Behavior Observation}

It was not possible to find a single extant robust observation instrument covering the Three-Ways. Instead, a bespoke tool was compiled, 


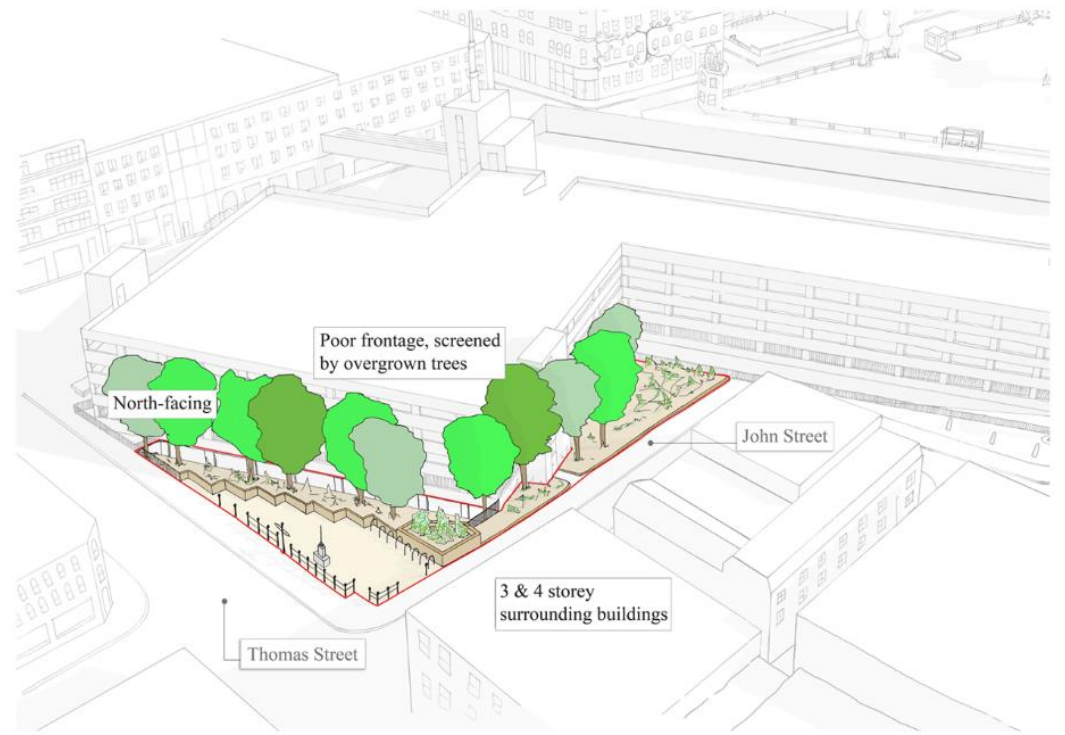

Figure 1 . The treatment space and boundary, before intervention. Source. Yeats (2012).

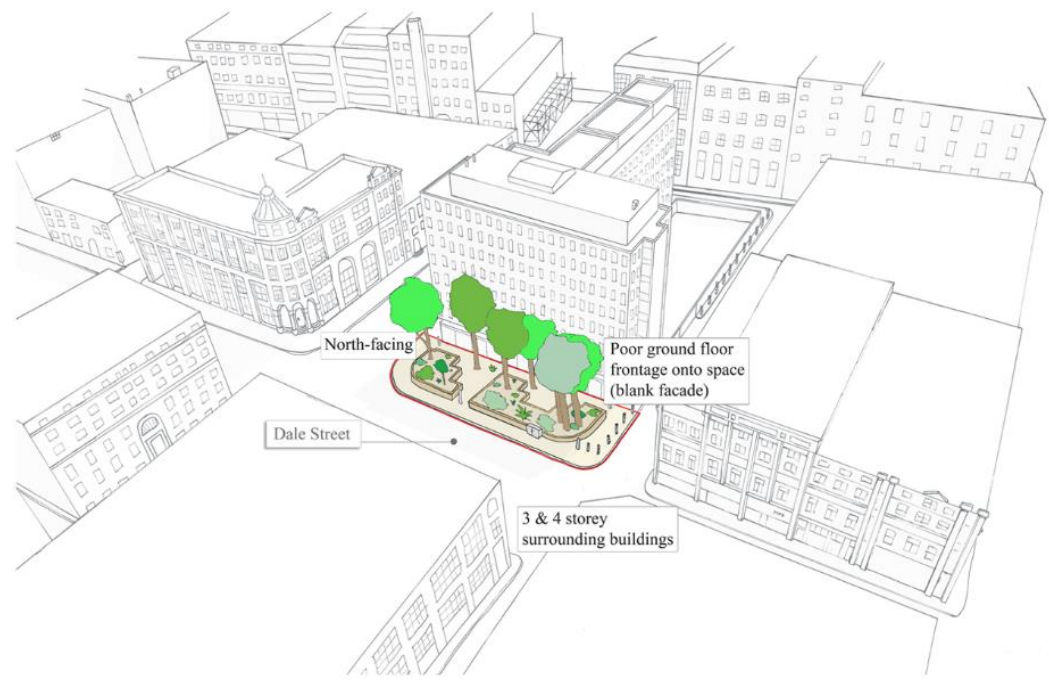

Figure 2. The control space and boundary.

Source. Yeats (2012). 
drawing on the strongest instrument available: System for Observing Play and Recreation in Communities (SOPARC), which is shown to be valid and reliable for physical activity in park settings. This tool provides an assessment of park users' physical activity levels, gender, activity types, estimated age, and ethnicity groupings (McKenzie \& Cohen, 2010).

SOPARC focuses on physical activity in the United States, and although it includes 28 categories, including non-physical activities, nine activity types were added for more comprehensive coverage of Connect and Take Notice behaviors (Appendix B). These additions included categories such as "talking and listening," taken from U.K. GOS (2008) example behaviors, together with pilot observation work carried out in both urban and suburban settings. Several physical activity codes such as "baseball" were removed, as they are not applicable to this particular U.K. setting.

In accordance with the Three-Ways objectives of the study, a departure from SOPARC was the exclusion of estimations of users' energy expenditure. Instead, emphasis was given to an alternative interpretation of primary and secondary activities. Within SOPARC, the most prominent physical activities are referred to as primary and the next most noticeable as secondary. To improve the capture of Connect and Take Notice activities, a secondary behavior from the same user was counted if that user was engaged simultaneously in more than one activity. The primary activity was regarded as the mode of movement or immobility (e.g., walking or sitting) and obvious additional activity as a secondary activity. For example, in the instance that a user was observed walking his or her dog and sharing a few words with another user, "dog-walking" would be coded as the primary activity and "talking and listening" the secondary behavior.

The main advantage of the secondary count was to capture activities that would be missed by SOPARC, including Be Active behaviors often paired with Connect activity. For example, children playing an informal ball game would be captured by SOPARC, whereas the fact that the children were talking and listening to one another might not be recorded. 
An additional departure from SOPARC was to record the duration of user activity, as it is considered an important component of public space vitality (Gehl, 2004; Mehta, 2007). Each observation session was $10 \mathrm{~min}$ long, which was split into four categories: $0 \leq 3 \mathrm{~min}$, $3<6 \mathrm{~min}, 6<9 \mathrm{~min}$, and 9+ $\min$. The majority of users' duration represented people passing through the space and therefore fell into the first category. At peak times, the number of people who stopped to use the space rarely exceeded eight to 10 users, allowing the researcher to keep approximate records of duration of stay.

A small pilot study tested new codes and implementation procedures, prior to the main study. To capture baseline measures before any intervention work was undertaken and given resource limitations, the pilot wok emphasized calibration of a priori, rather than inter-rater reliability (IRR). In particular, this involved several days of development of aspects of the protocol that were not taken from SOPARC. However, practicing of protocol involved testing of percentage agreement with a colleague from the same institute. Following two iterations of coding resolution between assessors, $89 \%$ agreement was found for primary behaviors and $80 \%$ for secondary behaviors. In light of project constraints, these figures were deemed acceptable to proceed with the main study.

In accordance with SOPARC protocol, observation data were collected at specified times in the morning, noon, afternoon, and evening. Observations were made at five specific intervals, for $10 \mathrm{~min}$ each time, including both busy times (e.g., weekday lunchtime) and quieter periods (e.g., mid-morning on weekdays) and made in both spaces at the same intervals on the same day. To decide which space to visit first, a coin was flipped before the start of each observation period. This brought a degree of randomization to the collection procedure, reducing the likelihood that results reflect a repeated time pattern in data collection.

The size of the behavior observation study was not based on formal power calculations. Instead, a sufficient number of observation periods were calculated based on pilot observations and careful estimates of persons expected, per observation session. This allowed 
approximate projections to be made about size of data set necessary to provide statistically meaningful insight (further detail is provided in the online supplemental appendix).

Coding entries were made into GoogleDocs via a digital tablet and a $3 G$ connection. Following Moore and Cosco (2010), printed plan copies of the spaces were used to record where Three-Ways took place in both spaces. All coding was made anonymously while sitting at the edge of the space, as if undertaking e-mails or surfing the Internet.

Prior to undertaking the field research, the ethics of the proposed mixed methods were discussed and an application was made to the Cambridge Psychology Research Ethics (CPRE) committee for a separate case study involving Global Positioning System (GPS) recorders. In accordance with CPRE guidance, ethical approval was not sought for this study, as it did not involve the following of individuals beyond the boundaries of the control and treatment spaces. Instead, a community was observed going about day-to-day life in two public spaces. Anonymity and privacy were protected as the coding and representation of data (behavior mapping) avoided specific individually identifying characteristics.

\section{Survey Data}

During the 42 days of the study ( 21 in 2011 and 2012), a sample of 212 users of the spaces completed a short questionnaire, approximately half in each space. Researchers approached people passing through or who had paused in the space, and invited them to answer a series of questions presented via the screen of a digital tablet. The researchers introduced themselves as undertaking university-led research on the quality of outdoor neighborhood provision and, post intervention, did not make reference to the site improvements. Participants were informed that the survey was anonymous and any participant questions regarding the study were answered by the researcher before proceeding with the survey. Responses were recorded into GoogleDocs via the tablet, and survey participants did not include previous participants of the workshop or volunteers involved in the implementation of physical improvements. 
The questions focused on users' momentary SWB and perceptions of the immediate physical and social environment. These questions and response scales were adopted or adapted from existing questionnaires, and the results are to be reported elsewhere. $A$ question of usage was also included within the survey. However, two researchers collected the survey data and the item was dropped from subsequent analysis because an inconsistent definition of usage was used when presenting the question to participants. The questionnaire concluded with an opportunity for respondents to express themselves in their own words, typed simultaneously by the researcher. The majority of users did not leave comments, and a balanced selection of positive, negative, and ambivalent comments were chosen. At their own discretion, participants reported approximately where they live (region and closest major road/landmark), whether they worked in the NQ, and their age group. Persons who lived and/or worked within the NQ boundary were considered to represent members of the local community.

\section{Data Management and Statistical Analyses}

Once data were exported from GoogleDocs into Microsoft Excel, it was opened in SPSS. To test the hypotheses, observation counts were split into three overarching categories: engaged, semi-engaged, and unengaged users (see online supplemental appendix). This process started with engaged users: people who stopped and engaged with the resources provided by the space. For example, a person who stood and looked at the public art, or sat and used a bench, rather than walking by without using the space in any tangible way. In contrast, an un-engaged user was a person who did not stop, or in any obvious way engage with the space. For instance, someone walking along the pavement, talking and listening to a companion but not interacting with the space, was considered to be un-engaged. Semi-engaged users represented people who did not stop but did engage with the space in a fleeting manner. For example, they walked past and glanced at children playing on the grass or at other users engaging with each other and the public art. Semi-engaged users were excluded from subsequent analyses, as they 
were not deemed to contribute sufficiently to the vitality of the space. Also, given the fleeting nature, the well-being impacts are likely to be most transient.

An independent statistician was consulted and several statistical tests considered. Given the exploratory nature of the study and the absence of sufficient individual-level covariate data, it was decided that the moderate additional information provided by complex analyses did not outweigh the benefits of a simple and direct approach. As such, each hypothesis was tested individually using Fishers Exact (FE) tests to establish statistical significance, and a standardized odds ratio calculation was used to provide an effect size. Care was taken to check violation of FE assumptions. In particular, the probability that the before (2011) and after (2012) samples were independent was considered to be high. For example, the city center has been shown to serve a large number of people across the City-Region of Greater Manchester (2.7 million), the North-West of England (7.1 million) and beyond (Harding, Marvin, \& Robson, 2006; ONS, 2011). In addition, the number of people who live and/or work locally has increased substantially between 2001 and 2011 (MCC, 2011). Overall, it was considered that the likelihood of the same people being observed in 2011 and 2012 was limited.

To prepare user counts to address the first research question, the number of engaged users was calculated by totaling the number of primary activities (numerator), in each space and in each year. The combined total of un-engaged users and semi-engaged users represented a denominator, providing a percentage when combined with the numerator. The totals were used within simple $2 \times 2$ analyses repeated as three separate FE tests for each of the five outcomes: First, to compare whether the proportion of engaged users observed within the control and treatment spaces was different at baseline; second, to assess statistical difference between the percentage of engaged users within the treatment space and control spaces, post intervention; and third, to compare before and after percentages of engaged users in both control and treatment spaces. An example analysis is provided within the online supplemental appendix. 
The data set was further analyzed for duration of activity and the Three-Ways. The grouping of Three-Ways activities was based on apriori definitions, and comparison was made between spaces and between years for each behavior category. Descriptive statistics revealed that a high proportion of users did not use the space for more than 3 min at baseline (2011), in the treatment, or the control space. This length of time was therefore used as a critical threshold, and the number of users categorized as staying longer than $3 \min (3<6 \mathrm{~min}, 6<$ $9 \mathrm{~min}$, and $9+\mathrm{min}$ ) were grouped, to make the same three comparisons above.

On average, $5 \%$ of the observation sessions were missing from the observation data set. Imputed calculations were not used, as the available data were sufficient to obtain statistically significant findings. IBM SPSS software was used for all statistical analyses.

\section{Results}

\section{The Sample}

The survey revealed that users of both spaces, in both 2011 and 2012, were largely people from Manchester and the North-West. ONS statistics show that people from these geographical areas are diverse, with a smaller proportion identifying themselves as White, compared with England in general (ONS, 2011). ONS data (2011) also show that economic inactivity in the city of Manchester is above the national average (36\% compared with $30 \%$ ). However, most other sociodemographic indicators are equivalent to English statistics (ONS, 2011), making this work suitable for some general insight for future application.

A total of 22,956 people were observed within the study, comprising of 5,141 in the control space and 6,005 in the treatment space, at baseline in 2011. After the intervention in 2012, 5,316 were observed in the control space and 6,494 in the treatment space. More people were observed in the treatment space because this was a marginally busier space. Results concentrate on engaged users, that is, those people deemed to have stopped and engaged with the resources provided by the space. The number of users who used the space for 
more than $3 \mathrm{~min}$ is presented before those who used the space to Connect, Be Active, and Take Notice of the immediate social or physical environment (see Table 1).

It was not possible to code gender and estimated age of all individuals observed. However, approximately $65 \%$ of those observed were successfully coded for both categories, and it was found that $10 \%$ more males were sighted than females in each of the groups. This discrepancy was approximately the same in each space. The estimated age of users was found to be similar in each space, but in both locations, very few children under 10 and adults above the age of 64 (5\%) were observed.

\section{Main Findings}

Behavior changes. The community intervention appeared to bring about both significant and substantial increases in the number of engaged users, who also stayed for longer (see Table 2). Between 2011 and 2012, there was a $230 \%$ increase in engaged users and an increase of $240 \%$ of the same people staying longer than $3 \mathrm{~min}$. Odds ratios demonstrate it was 3.5 times more likely to witness users engaged with the new public space resources and 1.2 times more probable to see people remain in the renewed space, for at least $3 \mathrm{~min}$.

The intervention was also followed by both significant and substantial increases in Three-Ways behaviors (see Table 2). Between 2011 and 2012, there was an increase of 394\% in Connecting and an increase of $648 \%$ in Taking Notice. The smaller increase in Keeping Active (23\%) represents children playing on the new grass, usually accompanied by a parent. Odds ratios showed it was 1.7 times more likely to observe Connect activities in the treatment space in 2012 than in the same space a year before. It was 3.5 times more likely to see people Taking Notice in 2012. These represent small and medium effect sizes, respectively.

By way of contrast, Table 2 shows the number of engaged users, 3-min minimum stays, and those who engaged in health behaviors halved in the control space between 2011 and 2012. It is not clear why these activities diminished. It may have been, in part, due to an increase 
in MCC Street Warden presence in 2012. This is consistent with an observable reduction in the number of young people using the control space to smoke and drink alcohol.

Table 1. Summary of the Number of Users Observed Within Each Space in Each Year and the Number Deemed to Be Engaged Users of the Space.

\begin{tabular}{lcccc}
\hline & Control space & Treatment & Control space & Treatment \\
& $(2011)$ & space (2011) & $(2012)$ & space (2012) \\
\hline Total no. of users observed & 5,141 & 6,005 & 5,316 & 6,494 \\
No. of engaged users & 465 & 355 & 346 & 1,169 \\
\% of engaged users & 9 & 6 & 7 & 18 \\
\hline
\end{tabular}

Table 2. Changes in Number of Engaged Users, 3-Min Stay and Health Behavior in the Treatment and Control Spaces, Between 2011 and 2012.

\begin{tabular}{lccccccc}
\hline & \multicolumn{3}{l}{ Treatment space } & \multicolumn{5}{c}{ Control space } \\
\cline { 2 - 8 } Outcome & Count & Count & $\%$ & Effect size (OR) & Count & Count & $\%$ \\
Engaged users & 2011 & 2012 & change & & 2011 & 2012 & change \\
Duration (3 min+) & 285 & 1,169 & $230^{*}$ & 3.5 (medium) & 465 & 241 & $-48^{*}$ \\
Connect & 77 & 380 & $394^{*}$ & 1.7 (small) & 252 & 116 & $-46^{*}$ \\
Be Active & 65 & 80 & 23 & 0.3 (no effect) & 2 & 1 & -50 \\
Take Notice & 77 & 576 & $648^{*}$ & 3.5 (medium) & 34 & 16 & $-47^{*}$ \\
\hline
\end{tabular}

Note. $\mathrm{OR}=$ odds ratio.

*Significant change $(p<.001)$.

Community usage. Engaged users of the spaces who participated in the survey and live in and/or work in the NQ were deemed to represent members of the local community. The survey showed that community use increased by $14 \%$ in the treatment space, from $25 \%$ in 2011 to $39 \%$ in 2012. Although this was not found to represent a statistically significant finding $(p>.05)$, this may reflect the limited survey sample size. Within the control space, the percentage of local engaged users reduced by 3 , from $36 \%$ to $33 \%$.

Where the changes occurred. To answer which specific improvements were associated with the largest changes, the locations of changes were 
mapped and analyzed. Each dot in Figures 3 and 4 is placed in the approximate location coded by the researcher at the time the sighting was made.

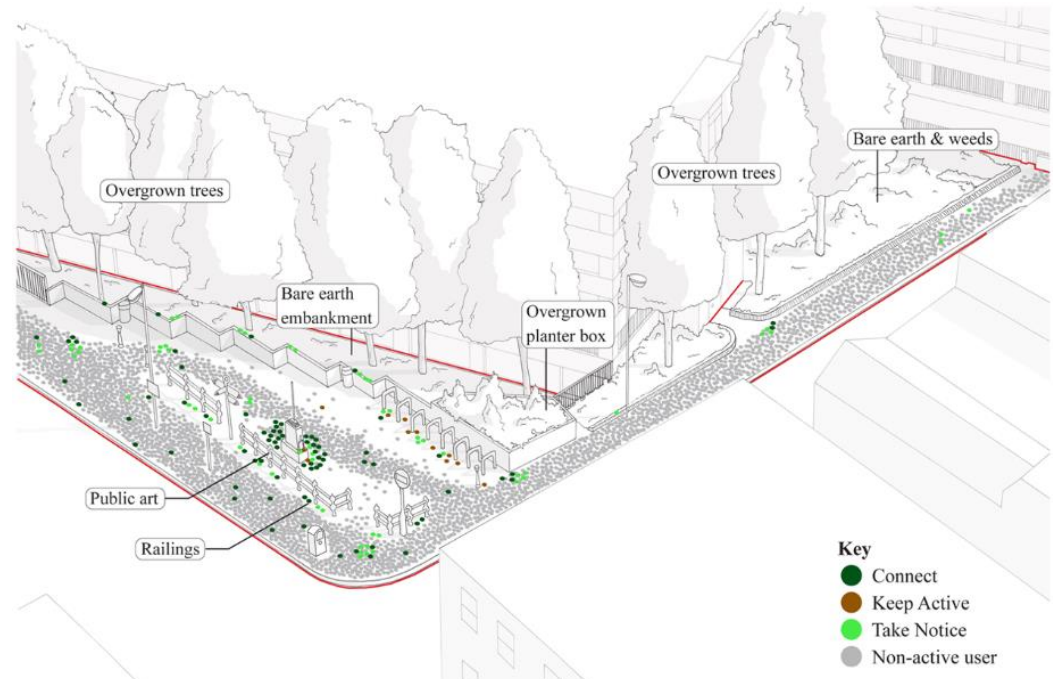

Figure 3. Treatment space in 2011, before community-led interventions. Source. Yeats (2012).

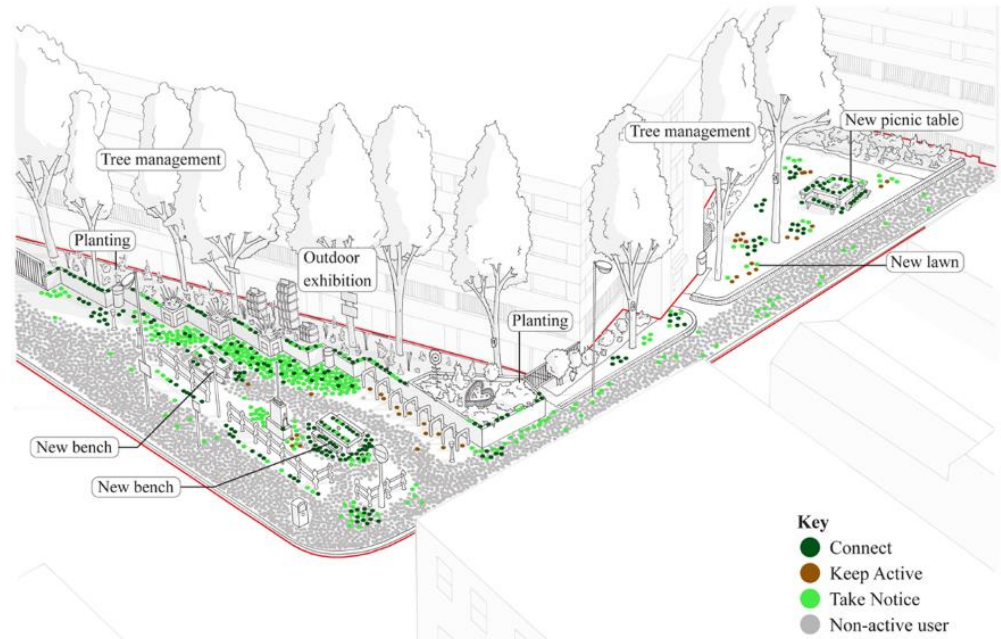

Figure 4. Treatment space in 2012, after community-led interventions. Source. Yeats (2012). 
These figures show that the largest changes in behavior corresponded with the outdoor exhibition space. For example, in Figure 4, the light green dots show the number of people who Took Notice of "Louse Vegas" and the public art. This is compared with Figure 3, which shows very few light green dots in the same space in 2011. The outdoor exhibition space also generated a large number of people connecting (dark green dots).

High levels of Connect and Take Notice activity are shown around the new picnic tables. Previous Connect patterns, such as along the wall at the rear of the paved areas or adjacent to railings, have been bolstered. Small clusters of Connecting and Taking Notice are found around the public art, bicycle racks, along the railing at the front of the space and, by the wall at the rear of the paved areas.

No activity was observed on the bare earth or overgrown areas under the trees in 2011. However, in 2012, sporadic indications of new Keep Active, Connect, and Take Notice activity can be seen on the new lawn areas and the previously overgrown planter box.

User comments: Post intervention. Approximately one third of the participants, who provided comments at the end of the survey, perceived several aspects of the treatment space to be negative. The majority of these remarks made reference to a critical aspect of the social environment. Throughout the 21-day observation period, a group of drunken users frequented the space. Most of the time, this group acted in a civilized manner and interacted with each other with little or no regard, for the people around them. However, the content of conversation that, at times, was relatively loud and brash, was perceived as "off putting" and "uncomfortable to be around."

This group, often referred to as "winos," could give the impression of "domination" and deter people from using the space. When the group interacted with others, it was often, although not always, to ask users for spare money, and in the event of rebuffed requests, participants described the group as "aggressive" and "cheeky with their begging." Instead of being attracted to the space to Connect with others and/or enjoy the public art, some users experienced 
nervousness and offense, and were repelled from using the space as a consequence. As well as being deterred from using the space, people did not always use the space as they would prefer.

The "winos" put me off using my laptop in the space. (Female Questionnaire Participant, 2012)

Although most participants made some sort of positive evaluation of the treatment space improvements, several qualified these comments with negative or critical appraisal. In the wake of public expenditure cutbacks, the Local Authority (MCC) cleaned the streets or collected bins once a week. Most qualified comments referred to the lack of "cleanliness and maintenance." One person described the cleanliness as "almost distracting to the public art." However, the majority of appraisals were balanced.

The space is not quite there, but it is a pleasant surprise. With further improvements, it would be a space I actively use more often. (Male questionnaire participant, 2012)

Respondents tended to identify something positive about either the physical or social environment, or both. In terms of improved functionality, people would mention the arrival of the benches that facilitated Connect and Take Notice activities. These new resources are likely to have indirectly supported experiences of individual well-being, such as happiness, and social well-being, such as belonging. An older gentleman referred to them as a "God-send" and was very grateful as he used them twice a week. Others referred to the benches as handy for eating lunch, without having to spend money and even an "incentive," given Manchester's general wet and "grey" weather conditions:

There are only 10 nice days a year (in Manchester), so it (the space) is a good incentive to get people out during the rest of the year! (Female questionnaire participant, 2012) 
Compared with a year before, the space was increasingly referred to as a "social" space, as more people used it, and was in turn good for Taking Notice as it promoted "people watching" and was "good for photography." People referred to Louse Vegas as humorous and absorbing and the artwork as a generally "really refreshing use of the space." The public space improvements therefore promoted social interaction, positive emotion, and short-term psychological functioning, such as a sense of being engaged. Several respondents referred to the interventions as imaginative and important because they reinforced the NQ's original "independent and arts based" identity, promoting social well-being locally and a "counter-culture" to increasingly commercial dynamics.

I love the quirkiness of Bug Hotels for the Northern Quarter; it counteracts the bland beer culture, which has developed recently. (Male questionnaire participant, 2012)

\section{Discussion}

This article outlined research involving mixed methods and an uncommon intervention that set out to establish whether low-cost changes to an outdoor neighborhood space could bring improvements in usage of the space and behaviors associated with well-being. We found that the implementation of small-scale public realm improvements significantly and substantially increased the number of users, their duration of stay, and well-being activities observed. We discovered that the proportion of community users increased by $14 \%$, suggesting an improvement in community life in the space. Survey comments also showed that several individuals reported using the space regularly, in particular the use of benches among older people and families. Although improvements were not comprehensive and contributed to some anti-social activity, overall, the impact was positive for both the vitality of the space and well-being activities.

The largest effect was found for an increase in users who engaged with the resources provided by the space generally. Increasing public space usage, without overcrowding, is deemed as contributing to 
the vitality, or liveliness of streets, and this study contributes to this achievement of this urban design objective. The same level of effect was found for users who Took Notice of their new physical and social environment. Figure 4, depicting behavior mapped in diagrammatic form, clearly reinforces these findings, demonstrating the success of the outdoor exhibition space. An increase in Connect activity was also substantial.

The Take Notice and Connect findings are useful preliminary insights because they are shown consistently to be key drivers of wellbeing (Chambers et al., 2009; Dolan et al., 2008; GOS, 2008; Helliwell \& Putnam, 2004; Hofmann et al., 2010; Krogh et al., 2011; Tang et al., 2012). Although a significant increase in physical activity was not found, the introduction of a larger area of grass and exercise apparatus may afford more adult and child usage in future designs.

The results suggest that a small pocket park, compared with larger parks or cycle network interventions (Hunter et al., 2015; Mayne et al., 2015), may be more beneficial for mental rather than physical health outcomes. Although, as found within a U.S. study, when perceived to be attractive among residents, nearby pocket parks can promote cost-effective walking compared with existing parks and playground space (Cohen et al., 2014). Most U.K. neighborhoods present frequent small-scale opportunities for intervention, and as exemplified, in principle, by the U.K. Behavioral Insights Team (BIT), the ability of small changes to make a big difference should not be underestimated (Halpern, 2015).

The ideas came from a small group of local community members and combined practical changes such as seating and WiFi with imaginative changes such as bug hotels. The changes were also implemented by a small group of local volunteers, which made the project very affordable. This is particularly noteworthy at a time when cuts to public space services and maintenance are continuing to take place in the United Kingdom and internationally. 


\section{Potential External and Confounding Influences}

Given the field-study nature of this work, it is important that we outline potential external confounding variables in this type of approach. First, there is the possibility that the weather or specific events could have affected changes in behavior uncovered. For example, high temperature is linked to higher levels of aggression and crime (Bell \& Fusco, 1989). Also, increased rainfall and extreme temperatures have been found to be consistently linked to decreased outdoor physical activity (Chan \& Ryan, 2009; Nikolopoulou \& Steemers, 2003; Ward et al., 2014). Fortunately for the study, average weather recordings are very similar in July 2011 and 2012 at the time of data collection, and because both sites were always observed on the same days and at similar times of day, daily weather fluctuations could also be ruled out as a potential confounder (see online supplementary material).

Throughout the project, events that could affect usage of the spaces were monitored via local media, the local community, and general observation. Such events include cultural festivals, major changes to transport or businesses, physical stressors (e.g., noise and pollution), and social stressors (e.g., crime and anti-social behavior). It is not clear whether the community-led engagement itself contributed to the increased usage, despite the curtailment of local media coverage. Future study may attempt to disentangle the potential relative contribution of each factor by comparing "top-down" interventions led by designers and policy makers with "bottom-up" interventions led by local communities and stakeholders.

The only notable difference found during the monitoring process was that criminal and anti-social activity was observed to decrease within the control space, and to increase in the treatment space between 2011 and 2012. It is unlikely that this involved the migration of negative behavior from the control space to the treatment space. It was observed that the latter involved an older age group, compared with a younger age group within the control space in 2011. As mentioned by several participants, the increased numbers of drunken citizens and the arrival of picnic benches in the treatment space brought about increased anti-social behavior. This influx of 
additional negative activity would almost certainly have reduced the magnitude of the intervention effect, so it is all the more remarkable that we found substantial benefits of the intervention.

\section{Comparison With Previous Research}

Our findings provide further evidence that quality of public space is linked to social use and liveliness (Gehl, 2004; Mehta, 2013). To the best of the authors' knowledge, this research has not been conducted with Take Notice behavior measures in the context of public space. Therefore, for this activity, comparison with previous research is indirect or not possible. Before and after, studies using objective measures to investigate links between Connect activity and urban design parameters are lacking (Talen \& Koschinsky, 2013). Qualitative research in New York has begun to explore social behavior in relation to WiFi in public spaces (Hampton, Livio, \& Goulet, 2010), but quantitative research is absent. Several of this study's public space improvements involved nature (e.g., new lawn, bug hotels). The positive influence of these changes is consistent with evidence that green spaces are linked with health benefits (Anderson et al., in press; Bowler, Buyung-Ali, Knight \& Pullin, 2010; Boyko \& Cooper, 2008; De Vries, 2010).

A small number of natural experiments have shown that improvements in public space quality can bring improvements in both reported and observed physical activity (Mayne et al., 2015; McCormack \& Shiell, 2011). These studies build on wider cross-sectional evidence, providing a suggestion of a causal relationship. However, they represent early findings and it is not clear that enhancement of public space brings substantial increases in activity (All-Party Parliamentary Group [APPG] on Well-Being Economics, 2014; Anderson et al., in press; Benton et al., in press; Burton, 2015; Hunter et al., 2015).

\section{Limitations and Key Areas of Future Development}

Our approach has significant advantages over more traditional methods. The study comprised clear a-priori hypotheses, monitoring of externalities, suppression of potential local media coverage, clear sampling criteria, and a well-matched control space. Data were 
collected before and after an intervention, including direct observations and users' verbal accounts of behavior and their experience. However, despite these achievements, four key areas of exploration remain.

1. Further development of observation measures. Although the observation tool used within the study gleaned key points of protocol from an established instrument (McKenzie et al., 2010), piloting of new aspects demonstrated high percentage agreement between raters, and the same researcher collected all data within the main study, validity and reliability are not clear. In particular, inter-rater reliability tests such as Cohen's Kappa are required within future research to improve agreement within the secondary behavior coding and to establish to what extent primary and secondary coding agreement occurred due to chance.

2. The use of follow-up measures. Future study would benefit from longer-term follow-up, post intervention. This will help to understand the role of novelty of public space changes promoting changes in behavior. For example, it is important that the efficacy of interventions does not "drop out" within relatively short periods of time, providing poor cost-benefit value for investors - both monetary and voluntary.

3. Complete inclusion of gender and age. In the absence of randomization, baseline characteristics are likely to systematically differ across intervention and control groups. Although descriptive statistics were undertaken with an incomplete data set, our study was unable to statistically test for differences. Researchers should seek age and gender data in particular, as these demographic characteristics are consistently correlated with physical activity. This will also permit multivariate with interrupted time-series analyses.

4. Same person repeated measures. Our study of the intervention is limited to correlational insights and therefore should not be interpreted as proven to produce the desired changes in behavior at an individual level. As such, there are important questions that should be empirically tested in future studies. 
For example, do the observed behaviors represent one-off or repeated activity? Were activities displaced from another part of the neighborhood or city? Also, where possible, further study should include repeated measures with the same individuals. This will improve the ability to predict outcomes regarding behavior changes and day-to-day lives of the individuals observed. Other potential improvements might involve repeated experience sampling methods coupled with objective measures such GPS recorders and Pedometers, in order that a stronger triangulation of findings may be achieved.

People who live and work in the city of Manchester are broadly representative of the United Kingdom in socio-demographic terms. Most people who visit the NQ are from the city, or the North-West of England, which is also similar to the United Kingdom's wider makeup (MCC, 2003; ONS, 2011). It is likely that the people observed are also reasonably representative of the U.K. population, although children and older people were under-represented, and males slightly overrepresented. The findings therefore have the most applicability for people between the ages of 10 and 64 . However, sample size does not permit extrapolation to the general population. Similar further research may help to avoid this shortcoming using full power calculations.

Furthermore, in line with recommendations (Benton et al., in press; Craig et al., 2012), future study would benefit from publishing a study protocol with a list of a-priori analyses, ahead of data collection.

\section{Conclusion}

Small-scale, low-cost urban design interventions, involving improvement in quality of local public space, had a positive impact on liveliness and two forms of behavior related to well-being, namely, Taking Notice and Connecting with others. To a lesser extent, there was also an increase in Keep Active behaviors among children. Behavior mapping shows that the public art and seating were among the most successful aspects of improvement. 
Since 2012, the Localism Act has been ratified in the United Kingdom, which is intended to empower local residents (Local Government Association [LGA], 2013). Drawing on this legislation, the insights offered here may be of practical value to communities seeking to improve local outdoor neighborhood space in the United Kingdom and elsewhere, particularly where similar democratic processes prevail. Alongside localism, international interest in well-being policy has increased, including in France, Canada, the United States, and Mexico (Legatum Institute, 2014). In the United Kingdom, there are APPGs on "Well-being Economics," "Mental Health," and "Mindfulness," as well as a "Commission on Well-Being and Policy." In addition, "What Works" centers for well-being and economic growth have been founded. Evidence on the economic (What Works Center for Local Economic Growth, 2014) and well-being activity effects of changes to the built and natural environment is urgently sought (APPG, 2014; Legatum Institute, 2014).

Our methodology demonstrated strong feasibility and represents an opportunity to further explore community-led public realm design as an effective population-level health intervention. This project responds to growing awareness and pressure related to evidence for well-being in the built environment. It shows how, in practice, urban planners and researchers may step toward goldstandard low-cost natural experiment evaluation of both urban design (e.g., street vitality) and well-being outcomes. The approach may also encourage local creativity, stewardship, and scientific curiosity to be harnessed. 


\section{Author Contributions}

Anderson facilitated the community design workshop, contributed to implementation of interventions, collected data, and led on study design, refinement of method, data analysis, data interpretation, and writing. Ruggeri, Steemers, and Huppert contributed to method, interpretation, writing, and general critical input.

\section{Acknowledgments}

We would like to thank Dr. Evangelos Kontopantelis from the University of Manchester's Centre for Biostatistics for his help with the approach to statistical analysis. We would also like to thank the Northern Quarter Greening Group for their sustained input in making the public space improvements happen, and happen on time. We also extend our gratitude to Leigh Yeats for his sketches of the control and treatment spaces used within the article.

\section{Declaration of Conflicting Interests}

The author(s) declared no potential conflicts of interest with respect to the research, authorship, and/or publication of this article.

\section{Funding}

The author(s) disclosed receipt of the following financial support for the research, authorship, and/or publication of this article: This research was supported by the Engineering and Physical Sciences Research Council (EPSRC) in the United Kingdom (Grant EP/P505445/1). 


\section{References}

Aked, J., \& Thompson, S. (2011). Five ways to wellbeing: New applications, new ways of thinking. London, England: New Economics Foundation. Retrieved from http://www.neweconomics.org/publications/entry/five-waysto-well-being-new-applications-new-ways-of-thinking Aked, J., Thompson, S., Marks, N., \& Cordon, C. (2008). Five ways to wellbeing: The evidence. London, England: New Economics Foundation. Retrieved from http://www.neweconomics.org/projects/entry/five-ways-towell-being

All-Party Parliamentary Group on Well-being Economics. (2014). Wellbeing in four policy areas. Retrieved from http://b.3cdn.net/nefoundation/ccdf9782b6d8700f7c_ Icm6i2ed7.pdf

Anderson, J., \& Baldwin, C. (in press). Building well-being: Neighborhood flourishing and tools for collaborative urban design intervention. In Handbook of community well-being (International Quality-ofLife series). London, England: Springer.

Anderson, J., Ruggeri, K., Steemers, K., \& Huppert, F. (in press). The need for a well-being science of cities: Findings from a multiple prize-winning neighborhood.

Ballas, D. (2013). What makes a happy city? Cities, 32(Suppl. 1), S39-S50. doi:10.1016/j.cities.2013.04.009

Bauman, A., \& Bull, F. (2007). Environmental correlates of physical activity and walking in adults and children: A review of reviews. London, England: National Institute for Health and Clinical Excellence. Retrieved from http://www.nice.org.uk/guidance/ ph8/evidence/environmental-correlates-of-physical-activityreview2 and https://www.researchgate.net/publication/254926580_Environ mental_Correlates_of_Physical_Activity_And_Walking_in_Adult s_and_Children_A_Review_of_Reviews

Bell, P. A., \& Fusco, M. E. (1989). Heat and violence in the Dallas field data: Linearity, curvilinearity, and heteroscedasticity. Journal of 
Applied Social Psychology, 19, 1479-1482. doi:10.1111/j.15591816.1989.tb01459.x

Benton, J., Anderson, J., Hunter, R. F., \& French, D. P. (in press). The effect of the built environment on physical activity behavior: A quantitative review of the risk of bias in natural experiments.

Bowler, D. E., Buyung-Ali, L. M., Knight, T. M., \& Pullin, A. S. (2010). A systematic review of evidence for the added benefits to health of exposure to natural environments. British Medical Commission: Public Health, 10, Article 456. doi:10.1186/14712458-10-456

Boyko, C., \& Cooper, R. (2008). The effect of the physical environment on mental wellbeing (Foresight project-mental capital and wellbeing). London, England: The Government Office for Science.

Branas, C. C., Cheney, R. A., MacDonald, J. M., Tam, V. W., Jackson, T. D., \& Ten-Have, T. R. (2011). A difference-in-differences analysis of health, safety, and greening vacant urban space. American Journal of Epidemiology, 174, 1296-1306.

Bronfenbrenner, U., \& Morris, P. (2007). The bioecological model of human development. In W. Damon \& R.M. Lerner (Eds), Child Psychology Handbook: Theoretical models of human development (pp.793-828). London, England: Wiley. doi:10.1002/9780470147658.chpsy0114.

Burton, E. (2015). Mental well-being and the influence of place. In H. Barton, S. Thompson, S. Burgess, \& M. Grant (Eds.), The Routledge handbook of planning for health and well-being: Shaping a sustainable and healthy future (pp. 150-161). Oxford, UK: Routledge.

Cattell, V., Dines, N., Gesler, W., \& Curtis, S. (2008). Mingling, observing, and lingering: Everyday public spaces and their implications for well-being and social relations. Health \& Place, 14, 544-561.

Centre for Disease Control and Prevention. (2015). Website summary.

Department of Health and Human Services, United States Departement of Health \& Human Services. 
http://www.cdc.gov/healthyweight/physical_activity/index.htm I.

Chambers, R., Gullone, E., \& Allen, N. B. (2009). Mindful emotion regulation: An integrative review. Clinical Psychology Review, 29, 560-572. doi:10.1016/j.

cpr.2009.06.005

Chan, C. B., \& Ryan, D. A. (2009). Assessing the effects of weather conditions on physical activity participation using objective measures. International Journal of Environment Research and Public Health, 6, 2639-2654. doi:10.3390/ ijerph6102639

Childs, M. C. (2004). Squares: A public place design guide for urbanists. University of New Mexico Press, Albuquerque, NM 87106

Cohen, D. A., Han, B., Pitkin Derose, K., Williamson, S., Marsh, T., \& McKenzie, T. L. (2013). Increasing physical activity in parks: Results of a randomized controlled intervention trial using community-based participatory research. American Journal of Preventative Medicine, 45, 590-597. doi:10.1016/j.amepre.2013.06.015.

Cohen, D. A., Marsh, T., Williamson, S., Han, B., Derose, K. P., Golinelli, D., \& McKenzie, T. L. (2014). The potential for pocket parks to increase physical activity. American Journal of Health Promotion, 28(suppl. 3), S19-S26. doi:10.4278/ajhp.130430QUAN-213

Coley, R. L., Sullivan, W., \& Kuo, F. (1997). Where does community grow? The social context created by nature in urban public housing. Environment and Behavior, 29, 468-494.

Cooper Marcus, C., \& Francis, C. (Eds.). (1998). People places: Design guidelines for urban open spaces (2nd ed.). Van Nostrand Reinhold publishers.

Craig, P., Cooper, C., Gunnell, D., Haw, S., Lawson, K., Macintyre, S., . . . Thompson, S. (2012). Using natural experiments to evaluate population health interventions: Guidance for producers and users of evidence. Journal of Epidemiology and Community Health, 66, 1182-1186. doi:10.1136/jech-2011-200375 
Department for Transport. (2013). Traffic Counts (Website providing street-level traffic data for every junction-to-junction link on the ' $A$ ' road and motorway network in Great Britain). Retrieved from www.dft.gov.uk/traffic-counts

De Vries, S. (2010). Nearby nature and human health: Looking at mechanisms and their implications. In C. Ward Thomspon, P. Aspinall, \& S. Bell (Eds.), Open space: People space 2 (pp. 7796). Oxford, UK: Routledge.

Dolan, P., Peasgood, T., \& White, M. (2008). Do we really know what makes us happy? A review of the economic literature on the factors associated with subjective well-being. Journal of Economic Psychology, 29:94-122. doi:10.1016/j.jeop.2007.09.001

Dombrowski, S. U., Knittle, K., Avenell, A., Araújo-Soares, V., \& Sniehotta, F. F. (2014). Long term maintenance of weight loss with non-surgical interventions in obese adults: Systematic review and meta-analyses of randomised controlled trials. British Medical Journal, 348, Article 2646.

doi:10.1136/bmj.g2646

Gehl, J. (2004). Public spaces, public life. The Danish Architectural Press. Government Office for Science. (2008). Foresight mental capital and well-being (Project final report). London, England: Author. Retrieved from https://www. gov.uk/government/uploads/system/uploads/attachment_data /file/292450/mental-capital-wellbeing-report.pdf.

Halpern, D. (2010). The hidden wealth of nations. Cambridge, UK: Polity Press.

Halpern, D. (2015). Inside the Nudge Unit: How small changes can make a big difference. London, England: WH Allen.

Hampton, K. N., Livio, O., \& Goulet, L. (2010). The social life of wireless urban spaces: Internet use, social networks, and the public realm. Journal of Communication, 60, 701-722. doi:10.1111/j.1460-2466.2010.01510.x

Harding. A., Marvin, S., \& Robson, B. (2006). A framework for cityregions-UK. Office of the Deputy Prime Minister. 
Health \& Social Care Information Centre. (2015). Statistics on obesity, physical activity and diet: England 2015.

Helliwell, J., \& Putnam, R. D. (2005). The social context of well-being. In F. A. Huppert, B. Keverne, \& N. Baylis (Eds.), The science of wellbeing (pp. 435-459). Oxford, UK: Oxford University Press. doi:10.1098/rstb.2004.1522

Hofmann, S. G., Sawyer, A. T., Witt, A. A., \& Oh, D. (2010). The effect of mindfulness-based therapy on anxiety and depression: A metaanalytic review. Journal of Consultant Clinical Psychology, 78, 169-183. doi:10.1037/a0018555

Holland, C., Clark, A., Katz, J., \& Peace, S. (2007). Social interactions in urban public places. Bristol, UK: Policy Press.

Hunter, R. F., Christian, H., Veitch, J., Astell-Burt, T., Hipp, J. A., \& Schipperijn, J. (2015). The impact of interventions to promote physical activity in urban green space: A systematic review and recommendations for future research. Social Science \& Medicine, 124, 246-256.

Huppert, F. A., \& So, T. T. (2013). Flourishing across Europe: Application of a new conceptual framework for defining well-Being. Social Indicators Research, 110, 837-861. doi:10.1007/s11205-0119966-7

Jacobs, J. (1961). The death and life of great American cities. New York, NY: Vintage Books.

Kaczynski, A. T., \& Henderson, K. A. (2007). Environmental correlates of physical activity: A Review of evidence about parks and recreation. Leisure Sciences: An Interdisciplinary Journal, 29, 315-354. doi:10.1080/01490400701394865.

Kellie, E. (2010). Rebuilding Manchester. Derby: DB Publishing. Krogh, J., Nordentoft, M., Sterne, J. A., \& Lawlor, D. A. (2011). The effect of exercise in clinically depressed adults: Systematic review and meta-analysis of randomized controlled trials. Journal of Clinical Psychology, 72, 529-538. doi:10.4088/JCP.08r04913blu

Kuo, B. C., \& Sullivan, W. C. (1998). Green common spaces and the social integration of inner-city older adults. Environment and Behavior, 30, 832-858. 
Lee, I. M., Shiroma, E. J., Lobelo, F., Puska, P., Blair, S. N., Katzmarzyk, P. T., \& Lancet Physical Activity Series Working Group. (2012). Effect of physical inactivity on major non-communicable diseases worldwide: An analysis of burden of disease and life expectancy. The Lancet, 380, 219-229. doi:10.1016/S01406736(12)61031-9.

Legatum Institute. (2014). Well-being and policy. London, England:

Commission on Well-being and Policy. Retrieved from http://li.com/programmes/the-commission-on-wellbeing-andpolicy

Local Government Association. (2013). Localism Act reviews. Retrieved from http://www.local.gov.uk/localism-act.

Lund, H. (2002). Pedestrian environments and sense of community. Journal of Planning Education and Research, 2, 3301-3312. Lynch, K. (1960). The image of the city. Cambridge MA: MIT Press. Maas, J., Dillen, S. M., Verheij, R. A., \& Groenewegen, P. P. (2009). Social contacts as a possible mechanism behind the relation between green space and health. Health \& Place, 15, 586-595.

Manchester City Council. (2003). Northern quarter development framework. Retrieved from www.manchester.gov.uk/./northern_quarter_development_fra mework.

Manchester City Council. (2011). Ward profiles for city centre and Ancoats and Clayton. Research and Intelligence Chief Executive's Department (Research and Performance). Marteau, T. M., Ogilvie, D., Roland, M., Suhrcke, M., \& Kelly, M. P. (2011). Judging nudging: Can nudging improve population health? British Medical Journal, 342, Article d228. doi:10.1136/bmj.d228.

Mayne, S. L., Auchincloss, A. H., \& Michael, Y. L. (2015). Impact of policy and built environment changes on obesity-related outcomes: $A$ systematic review of naturally occurring experiments. Obesity Review, 16, 362-375. doi:10.1111/ obr.12269. 
McCormack, G. R., \& Shiell, A. (2011). In search of causality: A systematic review of the relationship between the built environment and physical activity among adults. International Journal of Behavioral Nutrition and Physical Activity, 8, Article 125. doi:10.1186/1479-5868-8-125.

McKenzie, T. L., Cohen, D. A., Sehgal, A., Williamson, S., \& Golinelli, D. (2010). System for Observing Play and Recreation in Communities (SOPARC): Reliability and feasibility measures. Journal of Physical Activity \& Health, 3. Retrieved from http://www.ncbi.nlm.nih.gov/pmc/articles/PMC2957838/ Mehta, V. (2007). Lively streets: Determining environmental characteristics to support social behavior. Journal of Planning Education and Research, 27, 165-187. doi:10.1177/0739456X07307947

Mehta, V. (2013). The street: A quintessential social public space. New York, NY: Routledge.

Moore, R. C., \& Cosco, N. G. (2010). Using behaviour mapping to investigate healthy outdoor environments for children and families: Conceptual framework, procedures and applications. In

C. Ward Thomspon, P. Aspinall, \& S. Bell (Eds.), Open space:

People space 2 (pp. 33-72). Oxford, UK: Routledge.

New Economics Foundation. (2016). Unpublished Five ways to wellbeing survey undertaken in 2015 and shared via e-mail correspondence.

Nikolopoulou, M., \& Steemers, K. (2003). Thermal comfort and psychological adaptation as a guide for designing urban spaces. Energy and Buildings, 35, 95-101. doi:10.1016/S03787788(02)00084-1

Office for National Statistics. (2011). Census data. London, England:

Retrieved from http://www.ons.gov.uk/ons/guidemethod/census/2011/census-data/index.html

Office for National Statistics. (2013). Measuring national well-being, personal well-being across the UK, 2012/13. ONS Statistical Bulletin. Retrieved from http://www.ons.gov.uk/ons/rel/wellbeing/measuring-national- 
well-being/personal-well-being-across-the-uk-;2012-13/sb-personal-well-being-across-the-uk-2012-13.html

Ogilvie, D., Anderson, J., Bull, F., Huppert, F., Jones, A., Mattocks, C., \& Panter, J. (in press). Findings from Relocation to New Environments (RENEW) UK feasibility and pilot study. National Institute for Health Research.

Oldenburg, R. (1981). The great good place. Berkeley. University of

California Press.

Organization for Economic Co-Operation and Development. (2014).

Better policies for better lives: Obesity update. Directorate for Employment, Labor and Social Affairs.

Paulson, S., Davidson, R., Jha, A., \& Kabat-Zinn, J. (2013). Becoming conscious: The science of mindfulness. Annals of the New York Academy of Sciences, 1303(1), 87-104. doi:10.1111/nyas.12203 Public Health England. (2013a). Health profile: Manchester. Retrieved from http://www.apho.org.uk/resource/item.aspx?RID=126930 Public Health England. (2013b). North West Mental Wellbeing Survey 2012/13 (PHE Publications Gateway No. 2013240). Retrieved from http://www.nwph.net/ nwpho/Publications/NW\%20MWB_PHE_Final_28.11.13.pdf. Sofi, F., Valecchi, D., Bacci, D., Abbate, R., Gensini, G. F., Casini, A., \& Macchi, C. (2010). Physical activity and risk of cognitive decline: A meta-analysis of prospective studies. Journal of Internal Medicine, 269, 107-117. doi:10.1111/j.1365-2796.2010.02281.x Strack, F., \& Deutsch, R. (2004). Reflective and impulsive determinants of social behavior. Personality and Social Psychology Review, 8, 220-247.

Talen, E., \& Koschinsky, J. (2013). The walkable neighborhood: A literature review. International Journal of Sustainable Land use and Urban Planning, 1, 42-63.

Tang, Y. Y., Yang, L., Leve, L. D., \& Harold, G. T. (2012). Improving executive function and its neurobiological mechanisms through a mindfulness-based intervention: Advances within the field of developmental neuroscience. Child Development Perspectives, 6, 361-366. doi:10.1111/j.1750-8606.2012.00250.x 
Tibbalds, F. (1992). Making people-friendly towns: Improving the public environment in towns and cities. Harlow, UK: Longman.

United Nations. (2016). Population division: Urban population (\% of total). Retrieved from http://unstats.un.org/unsd/demographic/sconcerns/densurb/ densurbmethods.htm.

Veitch, J., Ball, K., Crawford, D., Abbott, G. R., \& Salmon, J. (2012). Park improvements and park activity: A natural experiment. American Journal of Preventative Medicine, 42, 616-619. doi:10.1016/j.amepre.2012.02.015

Ward, P., McKenzie, T. L., Cohen, D., Evenson, K. R., Golinelli, D., Hillier, A., ... Williamson, S. (2014). Physical activity surveillance in parks using direct observation. Preventative Chronic Diseases, 11, Article 130147. doi:10.5888/ pcd11.130147

Wendel-Vos, W., Droomers, M., Kremers, S., Brug J, \& Van Lenthe., F. (2007). Potential environmental determinants of physical activity in adults: A systematic review. Obesity Review, 8, 425440. doi:10.1111/j.1467-789X.2007.00370.x

What Works Centre for Local Economic Growth. (2014). Public realmBriefing paper. Retrieved from http://whatworksgrowth.org/wp-content/uploads/2014/11/ 14-11-20-Public-Realm-Briefing.pdf.

What Works Well-Being. (2016, April). Communities evidence program [Newsletter].

Whyte, W. (1980). The social life of small urban spaces. Washington, DC: Conservation Foundation.

Wood, L., Shannon, T., Bulsara, M., Pikora, T., McCormack, G., \& GilesCorti, B. (2008). The anatomy of the safe and social suburb: An exploratory study of the built environment, social capital and residents' perceptions of safety. Health \& Place, 14(1), 15-31. doi:10.1016/j.healthplace.2007.04.004

\section{Author Biographies}


Jamie Anderson's research investigates the built environment in relation to well-being. Previously, he worked for 6 years as an urban design consultant. He is currently a knowledge transfer fellow based in BuroHappold engineers while supervised by the Department of Architecture at Cambridge University.

Kai Ruggeri has a PhD in psychology and is a chartered psychologist. His roles include research associate at Cambridge Engineering Design Centre, director of a Junior Researcher Programme, and psychometrician at the Cambridge Psychometrics Centre.

Koen Steemers is previous head of the Department of Architecture at Cambridge University (2008-2014), currently head of Graduate Studies, and a director of Cambridge Architectural Research Ltd. His work deals with the built environment implications of energy use, human comfort and well-being.

Felicia Huppert is founder and director of the Cambridge Well-Being Institute, and emeritus professor of psychology, Department of Psychology, University of Cambridge. She is a fellow of the British Psychological Society and also Darwin College, Cambridge. 